\section{Responding to COVID-19 in Hong Kong}

\author{
Kelvin KW To ${ }^{1,2}, \mathrm{MD}, \mathrm{KY}$ Yuen $^{1,2}$ *, MD \\ ${ }^{1}$ Department of Microbiology, Li Ka Shing Faculty of Medicine, The University of Hong Kong, Hong Kong \\ 2 Department of Microbiology, Queen Mary Hospital, Hong Kong
}

*Corresponding author: kyyuen@hku.hk

Hong Kong Med J 2020;26:164-6

https://doi.org/10.12809/hkmj205101

The 2019 coronavirus disease (COVID-19) is caused by severe acute respiratory syndrome (SARS) coronavirus 2 (SARS-CoV-2). ${ }^{1}$ The COVID-19 is primarily an acute viral respiratory disease which can manifest as acute upper or lower respiratory tract syndrome of varying severity, from asymptomatic virus shedding, rhinorrhoea, sore throat, conjunctivitis to cough, asymptomatic or silent hypoxia, chest discomfort, respiratory failure, or even multiorgan failure. ${ }^{1,2}$ Extrapulmonary manifestations include diarrhoea, lymphopenia, thrombocytopenia, deranged liver and renal function, rhabdomyolysis, anosmia, dysgeusia, meningoencephalitis, GuillainBarre syndrome, Kawasaki disease like multisystem vasculitis, and thromboembolism. ${ }^{3-9}$ The outcome of COVID-19 is largely affected by older age and the presence of obesity and other underlying comorbidities. ${ }^{10,11}$ The crude fatality varies widely for different geographical regions from $0.4 \%$ to $10 \%{ }^{12,13}$

Despite over 6 million COVID-19 cases and 360000 deaths globally, Hong Kong has a total of about 1094 cases at the time of writing, which is one of the lowest per million population among developed regions. The painful experience of the SARS outbreak in 2003 sparked a large body of local animal surveillance, which showed that 39\% of Chinese horseshoe bats could be harbouring bat SARS-related coronaviruses. ${ }^{14}$ Knowing that coronaviruses are prone to genetic mutations and recombination which produce new virus species, and the presence of a large reservoir of SARS-related coronaviruses in these horseshoe bats, together with the culture of eating exotic mammals in southern China, Hong Kong has anticipated and prepared for the re-emergence of SARS and other novel viruses from animals since $2007 .^{15}$

Based on soft intelligence that an epidemic due to a suspected SARS-related coronavirus was looming in Wuhan on 31 December 2019, border thermal scanning and consensus reverse transcription polymerase chain reaction (RT-PCR) assays for unexplained pneumonia were started. The serious response level was activated by Centre for Health Protection on 4 January 2020. The University of Hong Kong-Shenzhen hospital has served as the sentinel for Hong Kong by identifying the first family cluster of COVID-19 who presented with symptoms after returning from Wuhan on 10 January $2020 .^{1}$
This family cluster allowed us to preliminarily validate our in-house test for SARS-CoV-2 before commercial test kits were available. This family cluster showed that COVID-19 can be acquired from hospital, spreads very efficiently in the family setting with six out of seven members affected, and can have mild or asymptomatic manifestations.

Hong Kong is at high risk for COVID-19 dissemination. It is among the most densely populated regions globally with at least 200000 people living in subdivided flats of 60 square feet or less. Furthermore, Hong Kong has a large elderly population with 1.27 million people over the age of 65 years who are susceptible to severe COVID-19. Hong Kong is also at high risk of travel-related case importation, as there are about 150000 people crossing the ShenzhenHong Kong border and about 200000 travelling via Hong Kong International Airport daily. Finally, Hong Kong has a cool dry winter which may favour virus transmission and its environmental stability. In view of the high number of mild or asymptomatic cases, the Hong Kong public was advised by medical colleagues from different medical specialties to practice universal masking in addition to good hand hygiene on 24 January 2020, despite some local dissenting views and opposite recommendation by the World Health Organization and overseas health authorities. The compliance of our community with face mask went up to $97 \%$ during the morning rush hour. ${ }^{16}$ It turned out that only $40 \%$ of our COVID-19 patients were locally acquired cases, and most local clusters of transmissions were related to maskoff activities. Thus, universal or community-wide masking, in addition to the standard border controls, case finding by extensive testing, mandatory admission for cases, rapid contact tracing and quarantine, and social distancing measures, may have given Hong Kong an edge in controlling the local spread of COVID-19. The high professional standard of Hong Kong healthcare workers, the excellent training in infection control, and the adequate supply of personal protective equipment have resulted in zero COVID-19-related mortality and morbidity among our hospital personnel 5 months after the pandemic began.

Epidemiological decisions must be made early enough to be effective, as transmission may have occurred 14 days before the case is detected. Thus, the 
first case from mainland China should immediately lead to land border control and quarantine of returnees. The first overseas case should lead to testing at the airport and quarantine of all overseas returnees. Increasing numbers of local clusters of untraceable sources should mandate more social distancing. But early case detection depends on extensive testing by RT-PCR especially for patients with mild symptoms. Extensive RT-PCR screening will continue to be one of the most important indicators guiding epidemiological decisions.

However, taking clinical specimens for RT-PCR by nasopharyngeal and throat swabbing of asymptomatic individuals induces discomfort and occasionally nasal bleeding. It may also induce coughing and sneezing, which endangers the healthcare workers. Mass screening would lead to a shortage of swabs. Hong Kong has circumvented these difficulties by patient self-collection of early morning posterior oropharyngeal (deep throat) saliva before breakfast and mouth rinsing. ${ }^{3,17}$ During sleep, the nasopharyngeal secretions of the upper respiratory tract will go posteriorly and pool around the oropharynx together with the bronchopulmonary secretions of the lower respiratory tract moved up by ciliary activity to almost the same level. Both upper and lower respiratory tract secretions are important for laboratory diagnosis because many patients have peripheral multifocal ground glass opacities on their lung computed tomography scan despite paucity of respiratory symptoms. If the patient can clear the throat by a coughing and gurgling manoeuvre at least 5 to 10 times into a sputum container with $2 \mathrm{~mL}$ of viral transport medium, the sensitivity would be similar if not better than the nasopharyngeal and throat swab. This is especially useful for daily viral load monitoring in antiviral treatment trial during which many patients resent the discomfort of taking daily nasopharyngeal swabs. ${ }^{18}$ With reliable collection of early morning posterior oropharyngeal saliva, the viral load of COVID-19 patients was found to peak early at the time of symptom onset or at presentation, or even before symptom onset during the period of quarantine.

Although mandatory admission of all RT-PCR positive patients, including those subclinical or mildly symptomatic, has led to a shortage of negative pressure single isolation rooms, this arrangement which is mandated by public health ordinance allows early recruitment of patients for antiviral therapy. Ex vivo lung tissue explant challenged by SARS-CoV-2 showed that the innate immune response of lung tissue by interferons and inflammatory cytokines/ chemokines were markedly suppressed. ${ }^{19}$ Studies of the SARS outbreak in 2003 showed that interferonbeta can be synergistic with ribavirin, and a combination of lopinavir-ritonavir and ribavirin can markedly improve the outcome of SARS patients in terms of mortality and respiratory failure. ${ }^{20,21} \mathrm{~A}$ recently published multicentre, prospective, openlabel, randomised, phase 2 trial showed that triple antiviral therapy (interferon beta-1b, lopinavirritonavir, and ribavirin) was safe and superior to lopinavir-ritonavir alone in alleviating symptoms and shortening the duration of viral shedding and hospital stay in adult patients with mild to moderate COVID-19. ${ }^{18}$ The early admission of patients for assessment, antiviral therapy, and respiratory support may explain our very low crude fatality rate of less than $0.4 \%$ in Hong Kong. Although remdesivir was also shown to reduce time to recovery in a large randomised control, this drug is unlikely to be readily available in Hong Kong as the production cannot meet the huge demand. ${ }^{22}$ Therefore we are collecting convalescent plasma from recovered patients with high serum neutralising antibody titre and use it as a salvage therapy for those who do not respond to antiviral treatment including interferon beta-1b or remdesivir.

Hong Kong cannot be complacent, because just one super-spreading event in Amoy Garden during the 2003 SARS outbreak led to an overloading and paralysis of our hospital service. Fortunately, such events have not happened yet for COVID-19. The emergency evacuations of residents from buildings with faulty sewage vent pipes were wakeup calls for our urgent attention to the maintenance of such sewage systems. The cluster of seven COVID-19 cases in Luk Chuen House at Lek Yuen Estate, living in six units on different floors, could herald a major super-spreading event and should not be treated simply as just one more community cluster. Extensive RT-PCR testing for at least one person per thousand population per day for any mild respiratory symptoms should be conducted in all 18 districts to minimise the evolvement of super-spreading events. The SARS-CoV-2 will continue to circulate during the summer and may cause an explosive outbreak in winter because our herd immunity is very low. Even the seroprevalence among Hong Kong returnees from Hubei is only $3.8 \% .{ }^{23}$ A safe and effective vaccine is unlikely to become widely available for another 12 months or more. Thus, SARS-CoV-2 will likely become another seasonal respiratory coronavirus circulating in humans for many years to come. More research on the animal source of SARS-CoV-2, pathogenesis and immunology, and effective control measures are urgently needed.

\section{Author contributions}

All authors contributed to the concept or design of the study, acquisition and analysis of the data, drafting of the manuscript, and critical revision of the manuscript for important intellectual content. All authors had full access to the data, contributed to the study, approved the final version for publication, and take responsibility for its accuracy and integrity. 


\section{Conflicts of interest}

All authors have disclosed no conflicts of interest.

\section{Funding/support}

The authors' studies were partly supported by donations from Richard Yu and Carol Yu, May Tam Mak Mei Yin, the Shaw Foundation Hong Kong, Michael Seak-Kan Tong, Respiratory Viral Research Foundation, Hui Ming, Hui Hoy and Chow Sin Lan Charity Fund, Chan Yin Chuen Memorial Charitable Foundation, Marina Man-Wai Lee, the Hong Kong Hainan Commercial Association South China Microbiology Research Fund, the Jessie \& George Ho Charitable Foundation, Perfect Shape Medical, and Kai Chong Tong; and by funding from the Consultancy Service for Enhancing Laboratory Surveillance of Emerging Infectious Diseases and Research Capability on Antimicrobial Resistance for the Department of Health of the Hong Kong Special Administrative Region Government; the Theme-Based Research Scheme (T11/707/15) of the Research Grants Council; Hong Kong Special Administrative Region; Sanming Project of Medicine in Shenzhen, China (no SZSM201911014); and the High Level-Hospital Program, Health Commission of Guangdong Province, China.

\section{References}

1. Chan JF, Yuan S, Kok KH, et al. A familial cluster of pneumonia associated with the 2019 novel coronavirus indicating person-to-person transmission: a study of a family cluster. Lancet 2020;395:514-23.

2. Hung IF, Cheng VC, Li X, et al. SARS-CoV-2 shedding and seroconversion among passengers quarantined after disembarking a cruise ship: a case series. Lancet Infect Dis. In press.

3. To KK, Tsang OT, Leung WS, et al. Temporal profiles of viral load in posterior oropharyngeal saliva samples and serum antibody responses during infection by SARS-CoV-2: an observational cohort study. Lancet Infect Dis 2020;20:565-74.

4. Cheung KS, Hung IF, Chan PP, et al. Gastrointestinal manifestations of SARS-CoV-2 infection and virus load in fecal samples from the Hong Kong cohort and systematic review and meta-analysis. Gastroenterology 2020 Apr 3. Epub ahead of print.

5. Guan WJ, Ni ZY, Hu Y, et al. Clinical characteristics of coronavirus disease 2019 in China. N Engl J Med 2020;382:1708-20.

6. Zhang Y, Xiao M, Zhang S, et al. Coagulopathy and antiphospholipid antibodies in patients with Covid-19. N Engl J Med 2020;382:e38.

7. Helms J, Kremer S, Merdji H, et al. Neurologic features in severe SARS-CoV-2 infection. N Engl J Med 2020 Apr 15. Epub ahead of print.

8. Tong JY, Wong A, Zhu D, Fastenberg JH, Tham T. The prevalence of olfactory and gustatory dysfunction in COVID-19 patients: A systematic review and metaanalysis. Otolaryngol Head Neck Surg 2020 May 5. Epub ahead of print.
9. Chung TW, Sridhar S, Zhang AJ, et al. Olfactory dysfunction in COVID-19 patients: observational cohort study and systematic review. Open Forum Infect Dis. In press.

10. Zhou F, Yu T, Du R, et al. Clinical course and risk factors for mortality of adult inpatients with COVID-19 in Wuhan, China: a retrospective cohort study. Lancet 2020;395:105462.

11. Kalligeros M, Shehadeh F, Mylona EK, et al. Association of obesity with disease severity among patients with COVID-19. Obesity (Silver Spring) 2020 Apr 30. Epub ahead of print.

12. Wu Z, McGoogan JM. Characteristics of and important lessons from the coronavirus disease 2019 (COVID-19) outbreak in China: Summary of a report of 72314 cases from the Chinese Center for Disease Control and Prevention. JAMA 2020 Feb 24. Epub ahead of print.

13. Ferrari R, Maggioni AP, Tavazzi L, Rapezzi C. The battle against COVID-19: mortality in Italy. Eur Heart J 2020 Apr 30. Epub ahead of print.

14. Lau SK, Woo PC, Li KS, et al. Severe acute respiratory syndrome coronavirus-like virus in Chinese horseshoe bats. Proc Natl Acad Sci U S A 2005;102:14040-5.

15. Cheng VC, Lau SK, Woo PC, Yuen KY. Severe acute respiratory syndrome coronavirus as an agent of emerging and reemerging infection. Clin Microbiol Rev 2007;20:66094.

16. Cheng VC, Wong SC, Chuang VW, et al. The role of community-wide wearing of face mask for control of coronavirus disease 2019 (COVID-19) epidemic due to SARS-CoV-2. J Infect 2020 Apr 23. Epub ahead of print.

17. To KK, Tsang OT, Chik-Yan Yip C, et al. Consistent detection of 2019 novel coronavirus in saliva. Clin Infect Dis 2020 Feb 12. Epub ahead of print.

18. Hung IF, Lung KC, Tso EY, et al. Triple combination of interferon beta-1b, lopinavir-ritonavir, and ribavirin in the treatment of patients admitted to hospital with COVID-19: an open-label, randomised, phase 2 trial. Lancet 2020;395:1695-704.

19. Chu H, Chan JF, Wang Y, et al. Comparative replication and immune activation profiles of SARS-CoV-2 and SARS-CoV in human lungs: an ex vivo study with implications for the pathogenesis of COVID-19. Clin Infect Dis 2020 Apr 9. Epub ahead of print.

20. Chu CM, Cheng VC, Hung IF, et al. Role of lopinavir/ ritonavir in the treatment of SARS: initial virological and clinical findings. Thorax 2004;59:252-6.

21. Chen F, Chan KH, Jiang Y, et al. In vitro susceptibility of 10 clinical isolates of SARS coronavirus to selected antiviral compounds. J Clin Virol 2004;31:69-75.

22. Beigel JH, Tomashek KM, Dodd LE, et al. Remdesivir for the treatment of Covid-19-preliminary report. N Engl J Med 2020 May 22. Epub ahead of print.

23. To KK, Cheng VC, Cai JP, et al. Seroprevalence of SARSCoV-2 in Hong Kong Special Administrative Region and our returnees evacuated from Hubei province of China: a multi-cohort study. Lancet Microbe. In press. 